\title{
Ocular Manifestations of Candidemia in Children
}

\author{
Julie L. Fierro, BA ${ }^{1}$, Priya A. Prasad, MPH ${ }^{1}$, Brian T. Fisher, DO, MSCE ${ }^{1,2,3}$, Jeffrey S. \\ Gerber, MD, PhD, MSCE ${ }^{1,2,3}$, Susan E. Coffin, MD, MPH ${ }^{1,2,3,5}$, Thomas J. Walsh, MD ${ }^{4}$, and \\ Theoklis E. Zaoutis, MD, MSCE $1,2,3,5$ \\ ${ }^{1}$ Division of Infectious Diseases, The Children's Hospital of Philadelphia, Philadelphia, \\ Pennsylvania, USA \\ ${ }^{2}$ Center for Pediatric Clinical Effectiveness, The Children's Hospital of Philadelphia, Philadelphia, \\ Pennsylvania, USA \\ ${ }^{3}$ Department of Pediatrics, The Children's Hospital of Philadelphia, Philadelphia, Pennsylvania, \\ USA \\ ${ }^{4}$ Transplantation-Oncology Infectious Diseases Program, Weill Cornell Medical Center of Cornell \\ University, New York, NY, USA \\ ${ }^{5}$ Center for Clinical Epidemiology and Biostatistics, University of Pennsylvania School of \\ Medicine, Philadelphia, Pennsylvania, USA
}

\section{Abstract}

Among 254 patients with candidemia who had a dilated retinal examination, 8 patients $(3.2 \%)$ were diagnosed with ocular disease resulting in retinal detachment in two patients and globe rupture in one patient. This study found that ocular candidiasis is an uncommon but serious sightthreatening complication in pediatric patients with candidemia.

\section{Keywords}

Candidemia; Candida; Ocular; Children

\section{Introduction}

Candida species are among the most frequent causes of blood stream infections, and can disseminate to any organ, including the heart, lung, brain, kidney, eye, liver, and spleen. Hematogenous spread of Candida to the eye can result in ocular disease and vision loss. Ocular lesions in patients with candidemia include chorioretinitis and endophthalmitis. According to the Infectious Diseases Society of America practice guidelines, a dilated ophthalmologic examination should be performed on all patients with candidemia to evaluate them for ocular involvement (1).

\footnotetext{
Corresponding Author: Theoklis E. Zaoutis, MD, MSCE, The Children's Hospital of Philadelphia, $34^{\text {th }}$ Street and Civic Center Boulevard, CHOP North, Suite 1527, Philadelphia, PA 19104, zaoutis@email.chop.edu, 267-426-5570, Fax: 267-426-0380. Publisher's Disclaimer: This is a PDF file of an unedited manuscript that has been accepted for publication. As a service to our customers we are providing this early version of the manuscript. The manuscript will undergo copyediting, typesetting, and review of the resulting proof before it is published in its final citable form. Please note that during the production process errors may be discovered which could affect the content, and all legal disclaimers that apply to the journal pertain.

Conflicts of Interest: T.E.Z has received research funding from Merck, Enzon, and Schering-Plough and has received speaking honoraria from Cephalon. All other authors: no conflict
} 
Despite the potential serious complications associated with ocular involvement, there is a paucity of data regarding the epidemiology and clinical characteristics of ocular candidiasis in children. Although the reported incidence of ocular candidiasis in the premature, lowbirth weight infant ranges from 0 to $40 \%(2-4)$, the incidence of ocular candidiasis within other pediatric age groups remains poorly defined due to limitations in sample size and a lack of disease-specific outcomes $(5,6)$. The aim of this study was to identify the incidence and outcomes of ocular disease in a large, population of pediatric patients with blood-culture proven candidiasis.

\section{Materials and Methods}

We conducted a retrospective cohort study of children with candidemia at The Children's Hospital of Philadelphia from January 1, 2000 through December 31, 2009 (7). The Children's Hospital of Philadelphia is an academic, tertiary care center with 489 beds and approximately 24, 000 hospital admissions per year.

Candidemia was defined by a blood culture yielding Candida spp. in a hospitalized patient. The date of infection onset was defined as the first day of a positive blood culture. If multiple episodes of candidemia occurred in the same patient during the study period, only the first episode was included in the analysis. Only children who survived $>7$ days after the diagnosis of candidemia were included in this study.

Structured chart review was performed to capture demographic and clinical characteristics of study participants. Abstracted data included age, sex, race, date of infection, and hospital location (e.g. neonatal intensive care unit, pediatric intensive care unit, general pediatric floor). All ophthalmology consult notes during the patient's hospital stay were reviewed for the presence of ocular disease. Ocular candidiasis was defined as either chorioretinitis (disease of the choroid or retina) or endophthalmitis (disease extending to the vitreous). The treatment regimens and outcomes were then reviewed for those patients with ocular candidiasis.

Statistical analysis was performed using STATA software (version 10). Frequency and proportions were determined for each variable.

\section{Results}

A total of 378 cases of candidemia were identified during the nine-year study period. Of these, 29 patients died within 7 days of the onset of candidemia; none of these patients received an eye examination before their death and all were excluded from further analysis. Of the remaining 349 patients, 254 (72.8\%) were examined by an ophthalmologist and received a dilated retinal examination (DRE). The median age and time to first eye assessment for patients was 2.5 years (Interquartile range (IQR): $0.52-11.9$ ) and 4 days (IQR: $3-7$ ), respectively.

Of the 254 patients examined, nine (3.5\%) were found to have abnormal DRE findings on the first ophthalmology examination. One additional patient had abnormal DRE findings on a second eye examination performed for persistent headaches and left orbital pain. Of these ten patients with abnormal findings, two were diagnosed with a non-infectious etiology attributed to their abnormal DRE processes. In total, of the 254 patients who had DRE, eight patients $(3.2 \%)$ had ophthalmologic involvement secondary to candidemia, including one neonate; four patients $(1.1 \%)$ were diagnosed with chorioretinitis and four $(1.1 \%)$ were diagnosed with endophthalmitis (Figure 1).The median age and time to first eye examination for patients with abnormal DRE's was 5.4 years (IQR: 3.0 - 13.4) and 5 days (IQR: 2.5-23), respectively. One patient, diagnosed with candidal endophthalmitis by ophthalmologic 
evaluation, had a vitreous biopsy; tissue pathologic studies and culture revealed no evidence of fungus.

The median duration of total therapy was 40 days (IQR: 22.75 - 63.5 days). Amphotericin B was administered to 7 of the 8 patients for initial treatment; 3 patients were treated with liposomal amphotericin B. The eighth patient received caspofungin for initial treatment. Among the 4 patients receiving monotherapy, one patient was treated with conventional amphotericin B and one with caspofungin; two patients were transitioned from conventional amphotericin B monotherapy to fluconazole monotherapy. Four patients received combination therapy: one patient received amphotericin B and flucytosine; a second received variable combinations of liposomal amphotericin $\mathrm{B}$, conventional amphotericin $\mathrm{B}$, voriconazole and caspofungin; a third was treated with liposomal amphotericin $\mathrm{B}$ for one day followed by fluconazole and caspofungin; the final patient, who had the vitreous biopsy, was treated with liposomal amphotericin or caspofungin monotherapy followed by amphotericin B, fluconazole and flucytosine with two courses of intravitreous injection of voriconazole.

Finally, three of the four patients (75\%) with endophthalmitis had additional eye complications; two patients developed retinal detachment, one of whom received caspofungin monotherapy, and the third had a globe rupture secondary to a deep corneal ulceration from Candida. The ultimate visual outcomes of these patients are unknown.

\section{Discussion}

We found that ocular candidiasis is an uncommon but serious sight-threatening complication in pediatric patients with candidemia. During the 10 -year study period, $3.2 \%$ of patients with positive blood cultures for a Candida spp. had associated findings on ophthalmologic examination consistent with ocular candidiasis, more than one-third of whom developed serious ophthalmologic complications. This incidence is comparable to other studies of children suggesting a lower incidence of ocular disease in children when compared with infants $(5,6,8-10)$.

The potential for serious complications associated with ocular candidiasis is highlighted by our results, as three of the four patients diagnosed with endophthalmitis developed sightthreatening complications. Other studies of pediatric patients have not reported complications secondary to ocular candidiasis. These poor outcomes underscore the importance of ophthalmologic examinations in all children with candidemia so that timely interventions can be performed when necessary.

The incidence of ocular involvement in other studies of neonates and older children with candidemia is comparable with that reported in this study $(5,6,8,9)$. Studies by Donahue et al. and Dozier et al. showed no episodes of ocular disease in cohorts of 30 patients ( 8 days 20 years old) and 83 patients ( 7 days -18 years old) with candidemia, respectively $(8,9)$. A study by Festekjian et al. documented two patients with ocular disease in a cohort of 167 patients ( 6 days -22 years old) with eye examinations following identification of candidemia (6). Another previous study documented that $8 \%$ of patients with candidemia $(0.6-14.3$ years old) had eye involvement (5). Variation in the incidence of ocular disease between studies may be explained by differences in the age of patient cohorts, underlying diagnoses, or other risk factors.

Studies have reported that the incidence of ocular involvement due to invasive candidiasis in infants ranges from $0-40 \%(2,3,10,11)$.The wide range of incidences of ocular disease in studies of infants suggests that this disease is more common in infants than in older children 
with candidiasis. Our findings may suggest a lower incidence of disseminated ocular disease in the pediatric population ( $>1$ year of age) when compared to infants.

This study has limitations. First, we had incomplete ascertainment of ocular candidiasis, including 29 patients who died before an ophthalmologic examination could be completed. If these patients were more likely to have suffered dissemination of Candida spp. to the eye, we may have underestimated the true incidence of ocular candidiasis in our cohort. Further, 95 patients who survived did not have an ophthalmologic examination. To minimize the impact of this incomplete ascertainment, we reviewed subsequent hospitalizations for surviving patient's that had not undergone ophthalmologic examinations and did not identify any symptom or sign consistent with ocular disease in this cohort. Finally, we were unable to investigate independent risk factors for ocular disease due to the limited number of events in this cohort of children with candidemia.

Given the complications associated with ocular candidiasis in pediatric patients, the impact of this disease cannot be overlooked. Clinicians should be encouraged to obtain ophthalmologic examinations on all patients with candidemia. Future multi-center studies should focus on identifying patients with candidemia that are at increased risk of ocular involvement. Earlier identification of ocular candidiasis with appropriate interventions may result in fewer complications and improved outcomes in children.

\section{Acknowledgments}

Sources of Support: This work was supported by the National Institutes of Health (1K23 AI0629753-01 to T.E.Z) and Merck Research Funding. This study was supported in part by the intramural research program of the National Cancer Institute.

\section{Abbreviations}

DRE Dilated Retinal Examination

\section{References}

1. Pappas PG, Kauffman CA, Andes D, et al. Clinical practice guidelines for the management of candidiasis 2009 update by the Infectious Diseases Society of America. Clin Infect Dis. 2009; 48:503-535. [PubMed: 19191635]

2. Lee BE, Cheung PY, Robinson JL, Evanochko C, Robertson CM. Comparative study of mortality and morbidity in premature infants (birth weight, $<1,250 \mathrm{~g}$ ) with candidemia or candidal meningitis. Clin Infect Dis. 1998; 27:559-565. [PubMed: 9770157]

3. Baley JE, Kliegman RM, Fanaroff AA. Disseminated fungal infections in very low-birth-weight infants: clinical manifestations and epidemiology. Pediatrics. 1984; 73:144-152. [PubMed: 6420764]

4. Baley JE, Annable WL, Kliegman RM. Candida endophthalmitis in the premature infant. J Pediatr. 1981; 98:458-461. [PubMed: 7205461]

5. Zaoutis TE, Greves HM, Lautenbach E, Bilker WB, Coffin SE. Risk factors for disseminated candidiasis in children with candidemia. Pediatr Infect Dis J. 2004; 23:635-641. [PubMed: 15247602]

6. Festekjian A, Neely M. Incidence and predictors of invasive candidiasis associated with candidaemia in children. Mycoses. 2011; 54:146-153. [PubMed: 19821906]

7. Zaoutis TE, Prasad PA, Localio AR, et al. Risk factors and predictors for candidemia in pediatric intensive care unit patients: implications for prevention. Clin Infect Dis. 2010; 51:e38-e45. [PubMed: 20636126]

8. Donahue SP, Hein E, Sinatra RB. Ocular involvement in children with candidemia. Am J Ophthalmol. 2003; 135:886-887. [PubMed: 12788131] 
9. Dozier CC, Tarantola RM, Jiramongkolchai K, Donahue SP. Fungal eye disease at a tertiary care center: the utility of routine inpatient consultation. Ophthalmology. 2011; 118:1671-1676. [PubMed: 21550121]

10. Noyola DE, Fernandez M, Moylett EH, Baker CJ. Ophthalmologic, visceral, and cardiac involvement in neonates with candidemia. Clin Infect Dis. 2001; 32:1018-1023. [PubMed: 11264029]

11. Benjamin DK Jr, Poole C, Steinbach WJ, Rowen JL, Walsh TJ. Neonatal candidemia and endorgan damage: a critical appraisal of the literature using meta-analytic techniques. Pediatrics. 2003; 112:634-640. [PubMed: 12949295] 


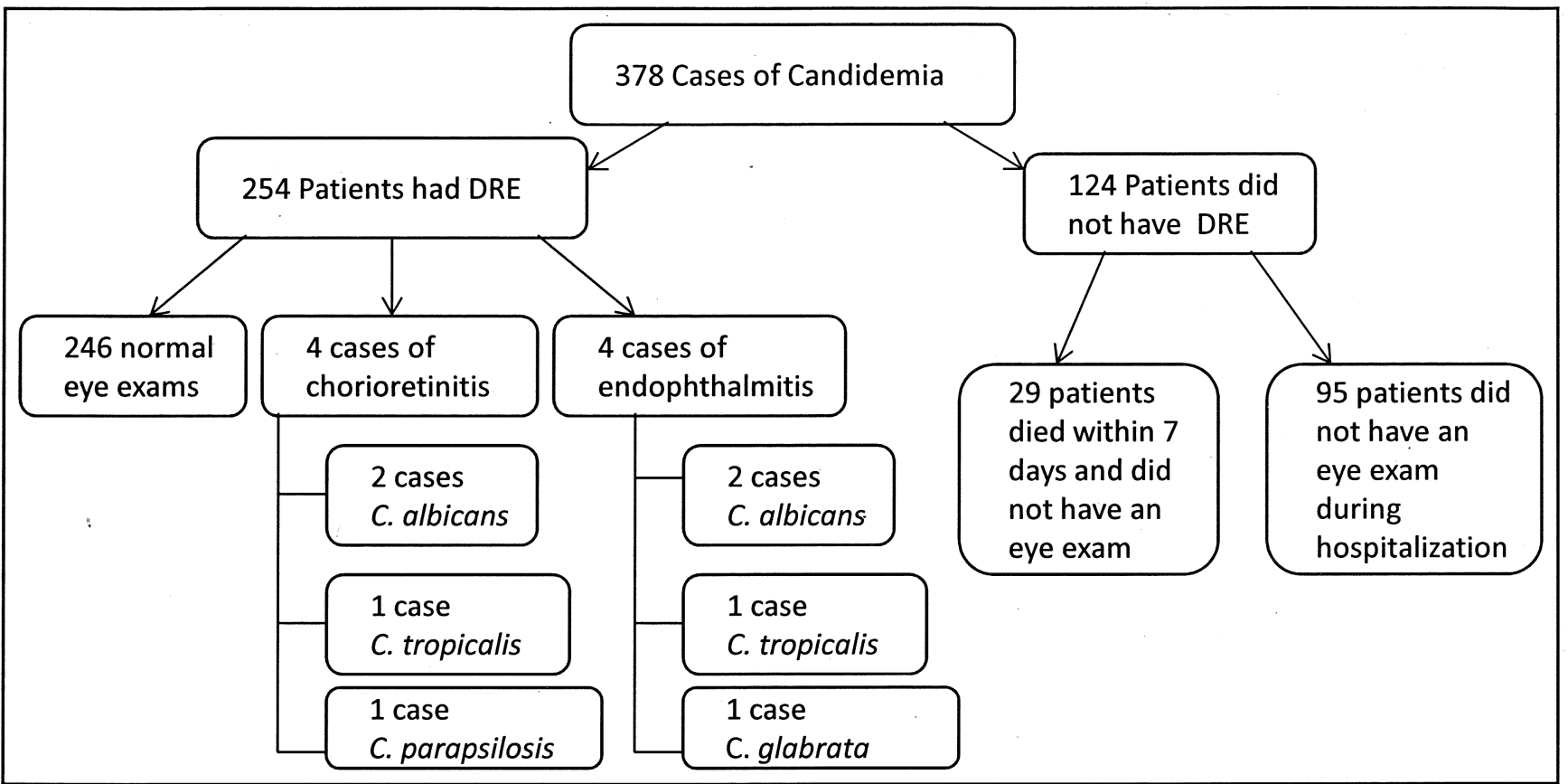

Figure 1.

Flow diagram of candidemia cohort showing the number of patients who did and did not receive an eye examination and the Candida species isolated from blood cultures of patients with abnormal eye examinations 